\title{
Review on Biodentine-A Bioactive Dentin Substitute
}

\author{
Priyalakshmi.S ${ }^{1}$, Manish Ranjan ${ }^{2}$ \\ ${ }^{1}$ Undergraduate student department of conservative dentistry, Saveetha Dental College,India \\ ${ }^{2}$ Reader department of conservative dentistry, Saveetha Dental College, India
}

\begin{abstract}
Quest for newer material are never ending especially in the field of dental science. Various materials have been formulated, tested, and standardised to obtain maximum benefits for good clinical performance. One such new material which was introduced is Biodentine which has numerous clinical applications. An ideal orthograde or retrograde filling material should seal the pathways of communication between the root canal system and its surrounding tissues. It should also be non toxic, non cariogenic, biocompatible, insoluble in tissue fluids, and dimensionally stable. Since existing materials did not have all these ideal characteristics, a new bioactive cement - Biodentine was introduced with all these ideal properties. It overcomes the drawbacks of Calcium hydroxide and Mineral trioxide aggregate. It is a promising material for repair of perforations of the pulpal floor, apexifications, open immature tooth, retrograde filling, pulp therapy. In this article the availability, composition, manipulation, mechanism of action, properties, clinical implications of biodentine has been reviewed.
\end{abstract}

\section{Introduction}

For many decades since 1928 calcium hydroxide has been standard material for maintaining the vitality of pulp since it is capable of stimulating tertiary dentin formation. However it has some drawbacks like poor bonding to dentin, material resorption ${ }^{[1] .}$ Later Mineral Trioxide Aggregate introduced by Torabinejad M, in 1990 is used as a material of choice for all dentinal defects due to their biocompatibility \& ability to induce calcium phosphate precipitate at interface to periodontium\& bone tissue repair. However there exists some drawbacks of this material such as slow setting kinetics and complicated handling properties ${ }^{[2]}$ The Minimal Intervention philosophy has seen a shift towards the biological non-operative management of teeth. Intervention when required, has become more effective and predictable with the advent and development of technologies to support this approach. One such material is biodentine, known as 'dentine in a capsule', a 'biocompatible and bioactive dentine substitute' which overcomes the draw backs of Calcium hydroxide and Mineral trioxide. It is quoted by Mark Hargreaves et al (2011) that biodentine allows a dentist to achieve biomimetic mineralisation within the depths of a carious cavity. Biodentine has a potential to revolutionise the management of the deep carious cavity in operative dentistry whether or not the pulp is exposed. Appreciable properties of biodentine includes good physical properties and its ability to stimulate tissue regeneration as well as good pulp response. Biodentine is a new Bio active cement with dentin like mechanical properties whic has beneficial effect on living cells \& acts in a bio compatible manner ${ }^{[3]}$.

\section{Availability}

Biodentine is a fine Hydrophilic powder composed of modified powder composition of MTA by addition of setting accelators and softners and a new predosed capsule formulation for use in a mixing device .All this largely improved the physical properties of this material making it much more user friendly (Wang et al 2008 Wonkornchaowalit and Lertchirakarn2011) Biodentine is available as powder in a capsule and liquid in a pipette

\section{Powder $^{[4]}[3.1]$}

\section{Composition Of Biodentine}

Tri-calcium silicate- This is the main core material.

Di-calcium silicate- this is the second core material

Calcium carbonate \& oxide- it acts as a filler.

Iron oxide-it acts as a colouring agent.

Zirconium oxide- it acts as a radioopacifier.

Liquid $^{[4]}[3.2]$

Calcium chloride- it acts as an accelerator.

Hydrosoluble polymer- it is a water reducing agent. 


\section{Manipulation}

The powder is mixed with the liquid in a capsule in the triturator for 30 seconds. Setting Time- approximately 12 minutes.

\section{Mechanism Of Action}

Biodentine induces mineralization after its application. Mineralization occurs in the form of osteodentine by expressing markers of odontoblasts \& increases TGF-Beta1 secretion from pulpal cells enabling early mineralization. During the setting of the cement Calcium hydroxide is formed. Due to its high $\mathrm{pH}, \mathrm{Calcium}$ hydroxide causes irritation at the area of exposure. This zone of coagulation necrosis has been suggested to cause division and migration of precursor cells to substrate surface; addition and cytodifferentiation into odontoblast like cells $^{\left[{ }^{[5]}\right.}$ Thereby Biodentine induces apposition of reactionary dentine by odontoblast stimulation and reparative dentin by cell differentiation ${ }^{[6]}$ Because of its high alkality it has inhibitory effects on microorganism.

\section{Properties}

Tissue Regeneration \& Early Mineralisation [6.1]-Biodentine induces early minerlization by increasing the secretion of TGF-61 from pulpal cells after its application ${ }^{[7]}$ It also acts by odontoblasts stimulation and cell differentiation, there by facilitating reactionary and tertiary dentin formation.Majorie et al(2012) did a study using biodentine, that it induces immortalized Murine Pulp Cell Differentiation into odontoblast like cells and stimulates bio mineralization. The study results suggested that biodentine is bioactive because it increased OD21 cell proliferation and it can be considered as a suitable material for clinical indications of dentine-pulp complex regeneration ${ }^{[.8]}$

Short setting time[6.2]- Biodentine sets within 12 minutes, which facilitates its use in immediate crown restoration (Tran et al., 2008) to make it directly intraorally "functional" without fear of the material deterioration. This can be applied in paediatric dentistry. ${ }^{[9]}$ A study by L Grech et al (2013) demonstrated that biodentine has a high wash out, low fluid uptake, resorption values, low setting time and superior mechanical properties. The addition of admixtures to tri calcium silicate-based cements affects the physical properties of these materials. ${ }^{[10]}$

Anti bacterial properties [6.3]- due to high alkaline $\mathrm{pH}$ Biodentine has inhibitory effect on the micro organisms. In addition, the alkaline change leads to the disinfection of surrounding hard and soft tissues. ${ }^{[11]}$

Bio compatibility[6.4] -Biodentine preserves pulp vitality and promotes its healing process ${ }^{[12]}$ Laurent et al tested a new Ca3SiO5- based material to evaluate its genotoxicity, cytoxicity and effects on the target cells specific function. The study concluded that the Biodentine material is biocompatible. The material was not found to affect the specific functions of the target cells and thus could safely be used ${ }^{[13]}$ About et al investigated biodentine activity by studying the effects on pulp progenitor cells activation, differentiation and dentine regeneration in the human tooth cultures. The study concluded that bio dentine is stimulating dentine regeneration by inducing odontoblast differentiation from pulp progenitor cells ${ }^{\left[{ }^{[14]}\right.}$ Laurent et al did further study to investigate the capacity of biodentine to affect TGF-61 secretion from pulp cells and to induce reparative dentine synthesis. Biodentine was applied directly onto the dental pulp in a human tooth culture model, resulting in a significant increase of TGF-61 secretion from pulp cells and thus inducing an early form of dental pulp mineralization shortly after its application. ${ }^{[15]}$ It does not affect human pulp fibroblast functions, expression of collagen1, dentine sialoprotein \& Nestin ${ }^{[16][17][18]}$. It is nongenotoxic.

Push Out Bond Strength Of Biodentine[6.5] - Biodentine has significantly higher push-out bond strength than MTA $(\mathrm{p}<0.5)$. The statistical ranking of push out bond strength values are DyractAP $>$ amalgam $\geq$ IRM $\geq$ Biodentine $>$ MTA. The push out bond strength of Dyract AP, amlgam, IRM and biodentine was not significantly different when immersed in sodium chloride, chlorhexidine and saline solution whereas MTA has lost its strength when exposed to chlorhexidine. Hence biodentine shows considerable performance as a perforation repair material even after being exposed to various endodontic irrigants. ${ }^{[19]}$

Good material handling[6.6] - ease of manuplation, better consistency, safety handling with favourable setting kinetics - about 12 minutes.Absence of post operative pain, when used as a dentin substitute in class $1 \&$ class 2 composite restorations. ${ }^{[20]}$

Specific properties of Biodentine as Dentin Substitute[6.7]

Elastic modulus, at $22.0 \mathrm{Gpa}$, is very similar to that of dentine at $18.5 ;{ }^{[21]}$

Compressive strength of about $220 \mathrm{MPa}$ is equal to average for dentine of $290 \mathrm{MPa}{ }^{\text {[21] }}$

Microhardness of Biodentin at $60 \mathrm{HVN}$ is same as that of natural dentin ${ }^{[21]}$

Acid resistance in acid erosion tests showed that the tricalcium silicate material presented with less surface disintegration. There was also deposition of apatite like calcium phosphate crystals on the surface. This shows improving interface between the dentine substitute Biodentine and the adjacent phosphate-rich hard tooth substance. ${ }^{[21]}$ 
Mariginal Adaptation and Sealing Ability[6.8] - The micromechanical adhesion of biodentine is caused by the alkaline effect during the setting reaction. This high $\mathrm{pH}$ causes organic tissues to dissolve out of the dentin tubule. The alkaline environment at the boundary area of contact between biodentine and hard tooth substance opens a path via which the dentin substitute mass can enter the exposed opening of the dentin canaliculi. This enables biodentine to be keyed to the dentine by means of innumerable microscopic cones, creating a stable anchorage with a sealing, bacteria-tight effect ${ }^{[21]}$

\section{Clinical Implications}

Pulp capping [7.1] - MTA has been proposed as a potential medicament for capping of pulps with reversible pulpitis because of its excellent tissue compatibility ${ }^{[22],[23] .}$. It is much superior to the routinely used calcium hydroxide based on the tissue reaction and the amount and type of dentin bridge formation ${ }^{[24]}$ Calcium hydroxide is associated with tissue necrosis and inflammation during the initial period of placement but no such inflammation or necrosis was seen in the pulp tissue adjacent to MTA. ${ }^{[25] .}$ Since there is no pulpal necrosis pulp tissue heals faster with MTA ${ }^{\text {,26] }}$ However MTA has its own draw backs. To overcome this biodentine can be used as pulp capping agent since it causes early mineralization by release of TGF -6 from pulpal cells to encourage pulp healing and by odontoblast stimulation for dentine bridge formation to protect the pulp. Alicja Mowicka et al (2013) did a study on the response of human dental pulp capped with Biodentine and MTA and reported that the majority of specimens showed complete dentinal bridge formation and absence of inflammatory pulpal response. Layers of well arranged odontoblast and odontoblast -like cells were formed to tubular dentine under the osteodentin. Therefore he concluded that within the limitations of his study biodentine had a good efficacy in the clinical setting and may be considered as an interesting alternative to MTA in pulp capping treatment during vital therapy. ${ }^{[27]}$

Repair of root perforations, apexification[7.2] - Due to poor bonding to dentin, material resorption and mechanical instability calcium hydroxide is not preferred for repair of root perforations, apexification, retrograde root filling,. So MTA is used most commonly in endodontics since 1990. But MTA has poor setting kinetics and poor handling properties. To overcome all these draw backs biodentine is used due to its appreciable properties like ease of handling, faster setting kinetics, biocompatibility, early mineralisation. (Dr. Francois Bronnec et al.,) ${ }^{28]}$

Root-End Filling[7.3]-Root end filing is one of the most important aspects of the periradicular surgery. The purpose of root end filling material is to establish an impermeable seal of all the apical avenues of the root canal system and prevent the percolation of bacteria and their products between the root canal systems and periradicular tissues. Many material have been used as root end filling agents but the main disadvantage is their failure to prevent leakage and the lack of biocompatibility. Requirements of ideal root end filling material is to adhere or bond to tooth tissue, be dimensionably stable, unaffected by moisture in either the set or unset state, be well tolerated by periradicular tissues with no inflammatory reactions, stimulate the regeneration of periodontium and be non toxic both systemically and locally ${ }^{[29][30][31]}$ Amalgam, although routinely used as root end filling material proved to be much inferior when tested with MTA ,since Zinc present in the amalgam is considered Cytotoxic ${ }^{[32][33]}$ Zinc oxide eugenol have been used in the past decades to replace amalgam; but they contain Eugenol which in contact with tissue fluids, is hydrolyzed and released ${ }^{[34}$ ]. Eugenol is the main cytotoxic component in the Zinc Oxide Eugenol cements ${ }^{[35][36]}$ Then came into existence the use of Glass Ionomer Cements as root end filling material . But it is greatly affected by moisture and blood during the initial setting time, resulting in increased solubility and decreased bond strength ${ }^{[37][38]}$ Later MTA was developed as a new root end filling material. The sealing ability, marginal adaptation of MTA was investigated .Good results were obtained with MTA when ranked with other materials ${ }^{[39]}$ But however it has a drawback of long setting time of about 45 minutes to 2 hours, so the material must be protected before they fully set ${ }^{[40] \text {. }}$ To overcome all this drawbacks Biodentine was introduced which preserves the pulp vitality and promotes its healing process. Biodentine stimulates dentine regeneration by inducing reparative dentine synthesis. Biodentine has better consistency, better handling, safety and faster setting time which creates no need for a two step obturation ${ }^{[41]}$

As dentine substitute [base] for posterior restorations [7.4]- Due to its dentine like mechanical properties Biodentine can be used as an dentine substitute ( Till Dammaschke et al., 2011) ${ }^{[42]}$ Since some of the previously used materials were not ideal. Those materials like Calcium hydroxide and Mineral trioxide aggregate have some disadvantages which are mentioned as follows. However Josette camalleri et al (2013) did a study using Biodentine as dentine replacement material. The results are obtained as, acid etching resulted in erosion of material surface with exposure of glass particles in the glass ionomer based materials. Biodentine exhibited a reduction in the chlorine peak and calcium silicon ratio when etched. Biodentine exhibited leakage both when it was etched and also when the surface was left unprepared. When used as a dentine replacement material in the sandwich technique over layered with composite, significant leakage occurred at the dentine to material interface ${ }^{[43]}$ So further studies are required to investigate on using the biodentine as dentine replacement material 


\section{Disadvantages Of Calcium Hydroxide}

Poor bonding to dentin ${ }_{\text {[44] }}$

Does not prevent microleakage in the long run ${ }^{[44]}$

The porosities ("tunnel defects") of the new formed hard tissue may act as a portal of entry for microorganisms ${ }^{[44]}$

Material Resorption and Mechanical instability. ${ }^{[44]}$

\section{Disadvantages Of Mta}

Discolouration potential-Iron and manganese have been mentioned as possible elements responsible for the discolouration tendency ${ }^{[45][46][47]}$

Difficult handling characteristic ${ }^{[48]}$

Long setting time -45 minutes to 2 hours, so the material must be protected before it is fully set ${ }^{[49]}$

High material cost (single use) -approximately 60-75 USD ${ }^{[50]}$

\section{Conclusion}

Disadvantages of calcium hydroxide and MTA has given a way for the use of biodentine with better results. Due to major advantages and appreciable properties and ability to achieve biomimetic mineralisation, biodentine has great potential to revolutionise the management of affected tooth in the operative dentistry and endodontics. However further studies are required to extend the future scope of this material regarding the clinical applications.

\section{References}

[1] Duda S, Dammaschike T. MaBnahmenzurVitalerhaltung der pulpa. GibtesAlternativenzumkalziumhydroxidbei der direktenuberkappung ? Quintessenz;59,2008,1327-1334, 1354.

[2] Tay et all 2007, Reyes-Carmona et al. 2009, Torabinejad\&parirokh 2010

[3] About I: Bioactivity of BiodentineTM: A Ca3Si05-based Dentine Substitute. Oral session, IADR Congress, Barcelona 2010.

[4] Till Dammaschke et al.THE dentist SEPTEMBER 2011;Direct pulping.

[5] Biodentine induces TGF-Beta 1 release from Human pulp cells and early dental pulp mineralization: DOI: 10.1111/j. 22 DEC 2011 1365-2591.2011.01995.x.

[6] Saidon et al., 2003 Shayegan et al., 2010.

[7] Laurent P, Camps J, About I,.Biodentine induces TGF-61 release from human pulp cells and early dental pulp mineralisation. Int Endod.J, 45(5),2010,439-48.

[8] Marjorie Zanihi, Jean Michel Sautier, Ariane Berdal, Stephane Simon.,Biodentine Induces Immortalized Murine Pulp Cell Differentiatiion into Odontoblast-like cells and Stimulates Biomineralisation. Journal of Endodontics.;38(9) Sep2012,1220-1226.

[9] Clinical evaluation of the performance and safety of a new dentine substitute, Biodentine, in the restoration of posterior teeth- a prospective study DOI 10.1007/s00784-012-0701-9.

[10] L.grech, B.Mallia, J.Camilleri.,Investigation of the physical properties of tricalcium silicate cement based root end filling materials. Dental Materials, 29(2), Feb2013, 20-28

[11] Dr.Med.Dent.Markus Th.Firla. ,Direct pulp capping with bioactive dentine substitute

[12] Septodent Biodentine scientific file,.2010.

[13] Laurent P,Camps J, De MEo M, Dejou J, About J,Induction of specific cell responses to a Ca3SiO5-based posterior restorative material. Dent Mater, 24(11),2008,1486-94.

[14] About I,Laurent P, Tecles,.Bioactivity of Biodentine a CA3SiO5-based Dentine Substitute.Oral session.IADR Congress July 2010,Barcelona,Spain.

[15] Laurent P,Camps J, About.I,.Biodentine induces TGF-61 release from human pulp cells and early dental pulp mineralisation. Int Endod J,45(5), 2010,,439-48.

[16] Pradelle-Plasse N, Tran X-V, Colon P, Laurent P, Aubut V, About I et al (2009) Emerging trends in (bio)material research. An Example of new material: preclinical multicentric studies on a new Ca3SiO5-based dental material. In: Goldberg M (ed) Biocompatibility or cytotoxic effects of dental composites, $1^{\text {st }}$ edn. Oxford, UK: Coxmoor Publishing Company,pp 184-203.

[17] Laurent P, Camps J, De Meo M, Dejou J, About I (2008) Induction of specific cell responses to a Ca(3)SiO(5)-based posterior restorative material. Dent Mater 24:1486-1494.

[18] Lesot H, Osman M, Ruch JV (1981) Immunofluorescent localization of collagens, fibronectins,\&laminin during terminal Differentiation of odontoblasts. DevBiol 82:371-381.

[19] Mehmet Burak Guneser,Makbule Bilge Akbulut,Ayce Unverdi Eldeniz.Effect of various endodontic irrigants on the Push-out-Bond Strength of Biodentine and Conventional Root Perforation Repair Materials.Journal of Endodontics,March2013;39(3)380-384.

[20] Briso AL, Mestrener SR, Delico G, Sunfeld RH, Bedran-Russo AK, de Alexandre RS et al (2007) Clinical Assessment of postoperative sensitivity in posterior composite restorations. Oper Dent 32:421-426.

[21] Direct pulp capping with a bioactive dentine substitute:Dr. Med. Dent. Markus Th. Firl.

[22] Eidelman E, Holan G, Fuks AB.Mineral trioxide aggregate vs formacresol in pulpotomized primary molars:a preliminary report.PediatrDent,23:15-18,2001.

[23] Bogen G,Kim JS, Bakland LK.Direct pulp capping with mineral trioxide aggregate. An observational study.JADA,139:305315,2008 .

[24] Chacko V, Kurikose S.Human pulpal response to mineral trioxide aggregate(MTA):A histologic study.J Clin Pediatr Dent,30:203209,2006

[25] Aeinehchi M, Eslami B, Ghanbariha M, Saffar AS.Mineral trioxide aggregate and calcium hydroxide as pulp-capping agents in human teeth:a preliminary report.Int Endod J,36:225-231,2003. 
[26] Accorinte M de 1,Holland R, ReisA,et al. Evaluation of mineral trioxide aggregate and calcium hydroxide cement as pulp capping agents in human teeth. J Endod,34:1-6,2008.

[27] Alicja Nowicka, Mariusz Lipski, Miroslaw Parafiniuk, Katarzyna Sporniak-Tutak, Damian Lichota, Anita Kosierkiewicz, Wojciech Kaczmarek, Jadwiga Buckzkowska-Radlinska. Response of human dental pulp capped with biodentine and mineral trioxide aggregate. Journal of Endodontics,june2013:39(6)743-747.

[28] BioDentine : A dentin substitute for the repair of root perforations, apexification and retrograde root filling. Dr Francois Bronnec DDS, MSc.

[29] Gartner AH, Dorn SO.Advances in endodontic surgery. Dent Clin North Am 1992;36:357-8.

[30] Kim S,Pecora G, Rubinstein RA, Dorcher, Kim J.Retrofilling materials and techniques.In:colour Atlas of Microsurgery in Endodontics,Ch.12.Philadelphia:WB Saunders,2001:115-24.

[31] Chong BS.A surgical alternative In:Managing Endodontic Failure in Practice,Ch.8.London;Quintessence Publishing Co.,Ltd,2004:123-47.

[32] Leiskar J.On the mechanism of cytotoxicity of silver and copper amalgams in a cell culture system.Scand J Dent Res 1974;82:7481.

[33] Leiskar J, Helgeland K.Mechanism of toxicity of dental materials. Int Endod J1981;14:42-49.

[34] Hume WR.In vitro studies on the local pharmacodynamics, pharmacology and toxicology of eugenol and zinc oxide eugenol. Int Endod J 1998;21:130-5.

[35] Hume W.Effect on respiration and division in human pulp, mouse fibroblasts and liver cells in vitro.J Dent Res 1984;63:1262-5.

[36] Koulaouzidou EA, Papazisis KT, Economides NA, Beltes P, Kortsaris AH. Antiproliferative effect of mineral trioxide aggregate,zinc oxide eugenol cement and glass ionomer cement against three fibroblastic cell lines. J Endod 2004;31:44-6.

[37] Germalmaz D, Yoruc B, Ozcan M, Alkumru HN. Effect of early water contact on glass ionomer luting cements. J Prothet Dent 1998;80:474.

[38] Suliman AA, Schulein TM, Boyer DB, Kohout FJ. Effect of etching and rinsing times and salivary contamination on etched glass ionomer cement bonded to resin composite. Dent Mater J 1989;5:171.

[39] Torabinejad M, Hong CU,McDonald F, Pitt Ford TR.Sealing ability of mineral trioxide when used as a root end filling material. J Endod 1993;19:591-5.

[40] Rufus Allwyn Meshack, K.Velkrishna,Pishipati, Vinayak Kalyan Chakravarthy, Jayashri Nerali. Overview of Root End Filling Materials.Int.Journal of Clinical Dental Science.March,2012;3(1):1-7.

[41] Rufus Allwyn Meshack, K.Velkrishna, Pishipati, Vinayak Kalyan Chakravarthy, Jayashri Nerali. Overview of Root End Filling Materials. Int.Journal of Clinical Dental Science.March2012;3(1):1-7.

[42] Koubi G. et al: A clinical study of a new Ca3SiO5-based material indicated as a dentine substitute. Abstract in clin Oral invest, Sevillia;2009.

[43] Josette Camilleri.Investigation of Biodentine as dentine replacement material. Journal of Dentistry.July2013;41(7)600-610.

[44] P.sangwan, A.sangwan, J.Duhan \& JA.rohilla:Tertiary dentinogenesis with calcium hydroxide:A review of proposed mechanism:International Endodontic journal;46,3-19,2013.

[45] Naik S,Hegde AM.Mineral trioxide aggregate as a pulpotomy agent in primary molars:an in vivo study. J Indian Soc Pedod Prev Dent 2005;23:13-6.

[46] Asgary S,Parirokh M, Eghbal Mj, Brink F.Chemical differences between white and gray mineral trioxide aggregate. J Endod 2005;31:101-3.

[47] Dammaschke T, Gerth HU, Zuchner H, Schafer E.Chemical and physical properties and bulk material characterization of white ProRoot MTA and two Portland cements. Dent Mater 2005;21:731-8.

[48] Masoud Parirokh,DMD,Ms, and Mahmound Torabinejad,DMD,MSD.PHD. Mineral trioxide aggregate:Comprehensive LiteratureReview-Part-3:Clinical applications, Drawbacks, and Mechanism of Action.JOE2010;36(3)400-413.

[49] Masoud Parirokh,DMD,Ms, and Mahmound Torabinejad,DMD,MSD.PHD. Mineral trioxide aggregate:Comprehensive LiteratureReview-Part-3:Clinical applications, Drawbacks, and Mechanism of Action.JOE2010;36(3)400-413.

[50] Srinivasan V, Waterhouse P, Whitworth P, Whitworth J.Mineral tioxide aggregate in paediatric dentistry. Int J Paediatr Dent,19:3447,2009 . 